\title{
Parâmetros ventilométricos e hemogasométricos em cadelas submetidas à ovário-histerectomia, pré-medicadas com tramadol ou morfina e anestesiadas com isofluorano*
}

\section{Ventilometric parameters and arterial blood gases in bitches undergoing ovariohisterectomy, pre-medicated with morphine or tramadol and anesthetized with isoflurane}

\author{
Paula Ferreira da Costa**, Juliana Vitti Moro, ${ }^{* * *}$ Edna Mireya Gómez Ortiz, ${ }^{* * *}$ Denise Granato Chung, ${ }^{* * * *}$ \\ Rodrigo de Lima Carneiro, ${ }^{* * *}$ Newton Nunes ${ }^{* * * * *}$
}

\begin{abstract}
Resumo
Avaliaram-se os efeitos nos parâmetros ventilométricos e hemogasométricos em cadelas submetidas à ovário-histerectomia (OHE), pré-medicadas com tramadol ou morfina e anestesiadas com isofluorano. Utilizaram-se 12 cadelas sem raça definida (SRD), divididas em dois grupos ( $n=6$ para cada grupo), todas com indução anestésica com propofol e manutenção com isofluorano em $\mathrm{O}_{2}$ a $100 \%$, sendo que a diferença entre os grupos se deu pela pré-medicação com tramadol (GT - 4mg/kg IM) ou morfina (GM - 0,4 mg/kg IM). As observações das variáveis iniciaram-se 30 minutos após a administração do bolus de propofol (M0). As demais colheitas dos dados foram realizadas em intervalos de 15 minutos, por um período de 60 minutos (M15, M30, M45 e M60, respectivamente). Não foram encontradas diferenças significativas entre os grupos ou momentos para as variáveis hemogasométricas, entretanto, as médias de $\mathrm{pH}$, déficit de base (DB) e pressão parcial de dióxido de carbono no sangue arterial $\left(\mathrm{PaCO}_{2}\right)$ ficaram fora da faixa normal para a espécie canina. Foram encontradas diferenças significativas para as variáveis: volume corrente (GM > GT de M0 a M60), pico de fluxo inspiratório e expiratório (GM > GT) e pressão de pico inspiratório, sendo que GM foi maior que GT em M0. Concluiu-se que ambos os opioides são seguros, não causando alterações importantes na ventilometria quando utilizados na pré-medicação em cadelas anestesiadas com isofluorano e submetidas à OHE, porém, a anestesia inalatória com isofluorano deve ser utilizada com cautela em animais com propensão à acidemia.
\end{abstract}

Palavras-chave: hemogasometria, opioides, ventilometria.

\begin{abstract}
We evaluated the effects on blood gas and ventilometric parameters in bitches undergoing ovariohysterectomy (OHE), pre-medicated with morphine or tramadol and anesthetized with isoflurane. We used 12 mongrel dogs, distributed into two groups ( $\mathrm{n}=6$ for each group), all with induction of anesthesia with propofol and maintained with isoflurane in $100 \% \mathrm{O} 2$, and the difference between groups was given by premedication with tramadol (GT - 4mg/kg IM) or morphine (GM - $0.4 \mathrm{mg} / \mathrm{kg}$ IM). The measurement of all variables was performed 30 minutes after propofol bolus (M0), and then, in intervals of 15 minutes, during 60 minutes (M15, M30, $\mathrm{M} 45$ and M60). No significant differences were found between groups or times for the gas variables, however $\mathrm{pH}, \mathrm{PaCO} 2$ and $\mathrm{DB}$ were outside the normal range for dogs. Significant differences were found for the variables: tidal volume (GM> GT M0 to M60), peak inspiratory and expiratory flow (GM> GT) and peak inspiratory pressure (GM was higher than GT in M0). It was concluded that both opioids are safe and will not cause major changes in ventilometry when used in premedication in dogs anesthetized with isoflurane and submitted to OHE, however, inhalation anesthesia with isoflurane should be used with caution in animals with a tendency to acidemia.
\end{abstract}

Keywords: blood gas, opioids, ventilometry.

\section{Introdução}

O controle da dor transoperatória tem importância em função da anestesia geral não eliminar todos os estímulos neuronais responsivos à dor (Lascelles et al., 1997). Nesse sentido, a analgesia preemptiva é uma estratégia para se obter analgesia trans e pós-cirúrgica eficiente, o que possibilita, além disso, diminuir significativamente a dose dos anestésicos requeridos para indução e manutenção, permitindo que o animal seja mantido em um plano anestésico ideal e minimizando o grau de depressão cardiorrespiratória.

*Recebido em 23 de setembro de 2013 e aceito em 25 de julho de 2014.

**Professora Dra da Fundação Educacional de Ituverava - Faculdade Dr. Francisco Maeda - Fafram, Ituverava - SP. Autor para correspondência: cidinhavet@ yahoo.com.br.

*** Médica-veterinária Autônoma.

****Aluno (a) Programa de Pós-Graduação em Cirurgia Veterinária da Faculdade de Ciências Agrárias e Veterinárias, UNESP - Câmpus de Jaboticabal.

*****Professor Adjunto do Departamento de Clínica e Cirurgia Veterinária, Faculdade de Ciências Agrárias e Veterinárias, UNESP, Câmpus de Jaboticabal. 
Atualmente, o uso de analgésicos, em particular os opioides, proporciona incremento na qualidade anestésica durante o período perioperatório. Seu emprego é justificado pela prevenção de alterações como arritmias, aumento no consumo de oxigênio pelo miocárdio, hipoventilação e hipóxia, decorrentes da ativação neuroendócrina causada por estímulos nociceptivos, relativos às lesões cirúrgicas (Gaynor, 1999)

O tramadol é um analgésico central que demonstra seletividade por receptores $\mu$ e também promove analgesia pela inibição da recaptação de noradrenalina e pelo aumento da liberação e inibição da recaptação de serotonina (Fantoni e Mastrocinque, 2002; Górniak, 2002; Bozkurt, 2005). Os parâmetros hemodinâmicos e respiratórios são pouco alterados por esse fármaco e o risco de depressão respiratória severa é pequeno quando comparado a outros opioides (Radbruch et al., 1996), sendo particularmente útil em pacientes com função cardiopulmonar comprometida (Grond e Sablotzki, 2004).

A morfina é o protótipo dos analgésicos opioides e correlatos, com a qual todos os outros fármacos desta classe são comparados (Fantoni e Mastrocinque, 2002). Trata-se de um agonista $\mu$, com bom efeito analgésico e que produz sedação moderada (Pascoe, 2000), sendo indicada no controle da dor moderada à severa (Fantoni et al., 2000). Esse fármaco deprime a respiração e isto se deve, provavelmente, a uma farta distribuição de receptores $\mu$ nas regiões bulbares. Essa depressão resulta no decréscimo do volume minuto respiratório e aumento na tensão de dióxido de carbono ao final da expiração $\left(\mathrm{EtCO}_{2}\right)$ (Thurmon et al., 1996). A morfina pode causar liberação de histamina (Veien et al., 2000) contribuindo para a hipotensão, taquicardia, broncoconstricção, colapso cardiovascular e prurido no período pós-operatório (Shepherd, 2003), sendo estes efeitos minimizados ou evitados caso a administração seja feita por via intramuscular ou subcutânea (Fantoni e Mastrocinque, 2002).

$\mathrm{O}$ isofluorano é um agente anestésico inalatório halogenado que possui efeitos cardiovasculares mínimos, com manutenção do débito cardíaco (DC) em valores fisiológicos nas concentrações de até 2 CAM (Steffey, 1996). Além disso, não sensibiliza o miocárdio às catecolaminas e por apresentar pouco efeito estimulante beta- -adrenérgico, diminui a resistência vascular periférica com consequente diminuição da pressão arterial (Amaral, 2001; Grief et al., 2003). Autores como Rezende et al. (2000), Gremião et al. (2003), Boscan et al. (2005), Doherty et al. (2007) e Pypendop et al. (2007) descreveram que a associação de fármacos como fenotiazínicos, opioides, dissociativos ou mesmo anestésicos locais podem contribuir para a diminuição da CAM do isofluorano, potencializando sua ação e diminuindo os efeitos adversos sobre os sistemas orgânicos .

Assim, com a finalidade de oferecer ao anestesiologista veterinário conhecimentos que permitam uma escolha segura da técnica anestésica, objetiva-se com este estudo avaliar o comportamento das variáveis ventilométricas e hemogasométricas em cadelas submetidas à ováriohisterectomia (OHE), pré-medicadas com tramadol ou morfina e anestesiadas pelo isofluorano.

\section{Material e métodos}

Este trabalho foi aprovado pela Comissão de Ética no Uso de Animais (CEUA) da Faculdade de Ciências Agrárias e
Veterinárias (FCAV) da Universidade Estadual Paulista (UNESP), campus Jaboticabal - SP, sob protocolo número 024178/12.

Foram utilizadas 12 cadelas, sem raça definida, com idade entre dois e sete anos, peso médio de $15 \pm 2,7 \mathrm{~kg}$, hígidas, provenientes do Projeto de Castração da cidade de Jaboticabal - SP, as quais foram submetidas ao procedimento de ováriohisterectomia (OHE), por meio da técnica padrão descrita por Stone et al. (1998), sendo realizada sempre pelo mesmo cirurgião. Elas foram divididas em dois grupos $(n=6$ para cada grupo); todas receberam indução anestésica com propofol (Propovan 1\% - Cristália - São Paulo, SP, Brasil) e mantidas em anestesia inalatória com isofluorano (Forane - Cristália São Paulo - SP) em oxigênio a $100 \%$, sendo que a diferença entre os grupos se deu pela pré-medicação, feita com tramadol (Tramadon $50 \mathrm{mg} / \mathrm{mL}$ - Cristália - São Paulo, Brasil) no grupo denominado GT, ou morfina (Dimorf $10 \mathrm{mg} / \mathrm{mL}$ - Cristália - São Paulo, Brasil) no grupo nomeado GM.

Os animais foram submetidos a jejum alimentar de 12 horas e hídrico de duas. Em seguida realizaram-se tricotomias das áreas sobre a artéria társica esquerda e a veia cefálica direita. Além disso, realizou-se tricotomia abdominal para o procedimento de OHE.

Antes do início da anestesia geral, todos os animais receberam, por via intramuscular, como única medicação pré-anestésica (MPA), tramadol, na dose de $2 \mathrm{mg} / \mathrm{kg}$ ou morfina na dose de 0,4 $\mathrm{mg} / \mathrm{kg}$, dependendo do grupo experimental ao qual as cadelas foram selecionadas (GT e GM, respectivamente).

Decorridos 20 minutos da MPA, as cadelas foram induzidas à anestesia geral pela administração intravenosa de propofol (Propovan 1\% - Cristália - São Paulo, SP, Brasil), na dose necessária à perda do reflexo laringotraqueal. A intubação foi realizada com sonda de Magill de diâmetro adequado ao porte do cão, a qual foi acoplada ao aparelho de anestesia inalatória com circuito anestésico com reinalação parcial de gases (OHMEDA - mod. Excel 210SE) instalado em linha com o filtro valvular, fornecendo-se $100 \%$ de oxigênio. Em seguida, os animais foram submetidos à anestesia inalatória com isofluorano, mantendo-se a concentração expirada em $2,1 \%$. Os pacientes foram mantidos em ventilação espontânea, sendo que o monitor de mecânica respiratória (Monitor DIXTAL - DX 8100 Intermed) teve seu sensor conectado à sonda orotraqueal dos animais para avaliação das seguintes variáveis: tensão de dióxido de carbono no final da expiração (ETCO2 - $\mathrm{mmHg}$ ), porcentagem da oxiemoglobina saturada por oxigênio (SpO2 - \%), frequência respiratória $(f-m p m)$, volume corrente inspirado $(\mathrm{Vti}-\mathrm{mL})$, tempo inspiratório (Tins - segundos), tempo expiratório (Texp segundos), isofluorano inspirado (Iso ins), pressão positiva ao final de expiração (PEEP - $\mathrm{cmH} 2 \mathrm{O}$ ), pressão de pico inspiratório ( $\mathrm{PIP}-\mathrm{cmH} 2 \mathrm{O}$ ), pressão média nas vias aéreas (MAP - $\mathrm{cmH} 2 \mathrm{O}$ ), trabalho respiratório $(\mathrm{W}-\mathrm{J} / \mathrm{L})$, produção de dióxido de carbono (VCO2 - $\mathrm{mL} / \mathrm{min}$ ), pico de fluxo inspiratório (PIF- L/min) e expiratório (PEF - L/min).

Ato contínuo, os animais foram posicionados em decúbito dorsal sobre colchão térmico ativo (GAYMAR-Mod. T-Pump) e no membro pélvico esquerdo, seguiu-se a cateterização percutânea da artéria társica dorsal, com Cateter Insyte 22G - BD Insyte $®$-Becton, Dickinson Indústria Cirúrgica- Juiz de Fora - MG- Brasil) de diâmetro adequado, o qual foi acoplado 
ao transdutor posicionado à altura do coração, para mensuração da pressão arterial média (PAM) e coleta das amostras de sangue $(0,3 \mathrm{~mL})$ para hemogasometria arterial (pressão parcial de oxigênio $\left(\mathrm{PaO}_{2}\right)$, em $\mathrm{mmHg}$; pressão parcial de dióxido de carbono $\left(\mathrm{PaCO}_{2}\right)$, em mmHg; saturação de oxi-hemoglobina $\left(\mathrm{SaO}_{2}\right)$, em \%; déficit de base (DB), em $\mathrm{mmol} / \mathrm{L}$; bicarbonato $\left(\mathrm{HCO}_{3}^{-}\right)$, em $\mathrm{mmol} / \mathrm{L}$ e $\mathrm{pH}$. As variáveis foram obtidas empregando-se equipamento específico (Hemogasômetro Roche OmiCRochi Diagnostics GmbH-Mannheim, Germany).

As observações das variáveis de interesse iniciaram-se 30 minutos após a administração do bolus de propofol (M0). As demais colheitas dos dados foram realizadas em intervalos de 15 minutos, por um período de 60 minutos (M15, M30, M45 e M60, respectivamente).

Imediatamente após a indução anestésica, todas as cadelas receberam fluidoterapia com solução de $\mathrm{NaCl}$ a $0,9 \%$ por via IV, na velocidade de infusão de $10 \mathrm{~mL} / \mathrm{Kg} / \mathrm{h}$, durante todo o protocolo experimental.

Para não haver interferência do procedimento cirúrgico na mensuração das variáveis, instituiu-se: o início da cirurgia imediatamente após M0, o pinçamento dos pedículos foi realizado 10 minutos após M15 e a ligadura do coto uterino 10 minutos após M45.

Os resultados tiveram seus valores submetidos ao teste da análise de variância (ANOVA) seguida do teste de Tukey quando as médias diferiram de forma significativa entre os momentos dentro do mesmo grupo e para a comparação das variações entre os grupos ( $p £ 0,05)$.

\section{Resultados}

De acordo com a Tabela 1, observam-se as variáveis ventilométricas. A análise estatística não revelou diferenças significativas entre grupos ou momentos para as variáveis $\mathrm{ETCO}_{2}, \mathrm{SpO}_{2}, f$, Tinsp, Texp, $\mathrm{VCO}_{2}, \mathrm{MAP}, \mathrm{PEEP}$. Entretanto, para $\mathrm{Vti}$, foram encontradas diferenças significativas entre grupos, sendo as médias de GM maiores que as de GT de M0 a M60. No que concerne à PIF e PEF, as médias do GM foram maiores que as de GT, sendo que para PIF, as médias de GM foram maiores que as de GT em M30 e M45 e para PEF as médias de GM foram maiores que as de GT de M15 a M60. Para a variável PIP, observou-se o contrário, onde a média de GT foi maior que a de GM apenas em M0, não havendo outras diferenças ao longo dos momentos entre os grupos.
Tabela 1: Valores médios e desvios padrão das variáveis ventilométricas em cadelas $(n=12)$ submetidas à ovariohisterectomia, pré-medicadas com tramadol (GT) ou morfina (GM) e anestesiadas pelo isofluorano

\begin{tabular}{|c|c|c|c|c|c|c|}
\hline \multirow[b]{2}{*}{ Parâmetros } & \multirow[b]{2}{*}{ Grupos } & \multicolumn{5}{|c|}{ Momentos } \\
\hline & & MO & M15 & M30 & M45 & M60 \\
\hline \multirow{2}{*}{$f(\mathrm{mpm})$} & GM & $15 \pm 7$ & $11 \pm 4$ & $14 \pm 6$ & $10 \pm 4$ & $11 \pm 4$ \\
\hline & GT & $16 \pm 9$ & $11 \pm 2$ & $10 \pm 2$ & $12 \pm 4$ & $12 \pm 4$ \\
\hline \multirow{2}{*}{$\mathrm{SpO}_{2}(\%)$} & GM & $98 \pm 0,8$ & $99 \pm 1$ & $98 \pm 0,8$ & $98 \pm 1$ & $98 \pm 1,4$ \\
\hline & GT & $98 \pm 1$ & $98 \pm 0,8$ & $98 \pm 0,5$ & $98 \pm 1$ & $98 \pm 0,6$ \\
\hline \multirow[b]{2}{*}{$\mathrm{ETCO}_{2}(\mathrm{mmHg})$} & GM & $46 \pm 2$ & $48 \pm 3$ & $50 \pm 3$ & $50 \pm 4$ & $52 \pm 4$ \\
\hline & GT & $43 \pm 4$ & $45 \pm 5$ & $49 \pm 7$ & $50 \pm 8$ & $47 \pm 6$ \\
\hline \multirow[t]{2}{*}{ Vti (mL) } & GM & $137 \pm 68^{A}$ & $\begin{array}{c}179 \pm \\
106^{\mathrm{A}}\end{array}$ & $162 \pm 92^{A}$ & $165 \pm 88^{A}$ & $170 \pm 92^{A}$ \\
\hline & GT & $124 \pm 36^{B}$ & $129 \pm 46^{B}$ & $130 \pm 50^{B}$ & $133 \pm 54^{B}$ & $131 \pm 49^{B}$ \\
\hline \multirow{2}{*}{ Tinsp (s) } & GM & $1,1 \pm 0,5$ & $1,1 \pm 0,7$ & $1,2 \pm 0,8$ & $2 \pm 1,3$ & $1,3 \pm 0,5$ \\
\hline & GT & $0,8 \pm 0,6$ & $1,2 \pm 1,3$ & $0,6 \pm 0,4$ & $1,2 \pm 1,3$ & $0,7 \pm 0,7$ \\
\hline \multirow{2}{*}{ Texp (s) } & GM & $4,3 \pm 3,2$ & $3 \pm 3$ & $3 \pm 3$ & $4 \pm 2,3$ & $5 \pm 3$ \\
\hline & GT & $1 \pm 0,9$ & $1,2 \pm 0,9$ & $0,9 \pm 0,6$ & $1,6 \pm 1,4$ & $1,1 \pm 1,2$ \\
\hline \multirow[b]{2}{*}{$\mathrm{PIP}\left(\mathrm{cmH}_{2} \mathrm{O}\right)$} & GM & $0,5 \pm 0,5^{A}$ & $0,7 \pm 0,8$ & $0,5 \pm 0,5$ & $1 \pm 0,9$ & $1,2 \pm 1,2$ \\
\hline & GT & $2,2 \pm 0,7^{\mathrm{B}}$ & $1,3 \pm 1$ & $1,3 \pm 1$ & $2 \pm 1,1$ & $2 \pm 1,1$ \\
\hline \multirow[b]{2}{*}{$\operatorname{MAP}\left(\mathrm{cmH}_{2} \mathrm{O}\right)$} & GM & $1 \pm 1$ & $1 \pm 1$ & $0 \pm 0$ & $1 \pm 1$ & $1 \pm 1$ \\
\hline & GT & $1 \pm 1$ & $1 \pm 1$ & $1 \pm 1$ & $1 \pm 1$ & $1 \pm 1$ \\
\hline \multirow[b]{2}{*}{$\operatorname{PEEP}\left(\mathrm{cmH}_{2} \mathrm{O}\right)$} & GM & $1 \pm 1$ & $1 \pm 1$ & $1 \pm 1$ & $1 \pm 1$ & $1 \pm 1$ \\
\hline & GT & $2 \pm 1$ & $1 \pm 1$ & $1 \pm 1$ & $1 \pm 1$ & $2 \pm 1$ \\
\hline \multirow{2}{*}{$\mathrm{W}(\mathrm{J} / \mathrm{L})$} & GM & $0 \pm 0$ & $0 \pm 0$ & $0 \pm 0$ & $0 \pm 0$ & $0 \pm 0$ \\
\hline & GT & $0 \pm 0$ & $0 \pm 0$ & $0 \pm 0$ & $0 \pm 0$ & $0 \pm 0$ \\
\hline \multirow{2}{*}{ Iso ins } & GM & $2,7 \pm 0,7$ & $3,2 \pm 0,6$ & $3,2 \pm 0,5$ & $2,7 \pm 0,4$ & $2,8 \pm 0,7$ \\
\hline & GT & $2,1 \pm 0,6$ & $2,4 \pm 0,8$ & $2,4 \pm 1$ & $2,1 \pm 1$ & $1,7 \pm 0,7$ \\
\hline \multirow{2}{*}{$\mathrm{VCO}_{2}(\mathrm{~mL} / \mathrm{min})$} & GM & $26 \pm 14$ & $32 \pm 20$ & $30 \pm 19$ & $34 \pm 17$ & $30 \pm 15$ \\
\hline & GT & $18 \pm 7$ & $18 \pm 8$ & $21 \pm 8$ & $24 \pm 13$ & $24 \pm 11$ \\
\hline \multirow{2}{*}{ PIF (L/min) } & GM & $11 \pm 3$ & $12 \pm 5$ & $12 \pm 5^{A}$ & $12 \pm 4^{\mathrm{A}}$ & $11 \pm 4$ \\
\hline & GT & $9 \pm 6$ & $6 \pm 6$ & $4 \pm 5^{B}$ & $4 \pm 4^{B}$ & $6 \pm 5$ \\
\hline \multirow{2}{*}{ PEF (L/min) } & GM & $11 \pm 2$ & $12 \pm 2^{\mathrm{A}}$ & $12 \pm 3^{A}$ & $13 \pm 2^{A}$ & $12 \pm 2^{A}$ \\
\hline & GT & $8 \pm 5$ & $6 \pm 5^{\mathrm{B}}$ & $4 \pm 5^{B}$ & $4 \pm 5^{B}$ & $6 \pm 6^{B}$ \\
\hline
\end{tabular}

Letras maiúsculas diferentes nas colunas indicam significância estatística, com $p<0,05$.

Pela Tabela 2, determinaram-se as variáveis hemogasométricas e a PAM. Relacionando-se à hemogasometria, nenhuma das variáveis estudadas demonstrou diferença estatística entre GM e GT ou dentro dos grupos, determinando a estabilidade desses parâmetros ao longo do período experimental, apesar de alguns parâmetros estarem com os valores fora da faixa 
considerada normal para a espécie. Para a PAM, observou-se diferença significativa apenas dentro do GM, sendo a média de M30 maior que M15.

Tabela 2: Valores médios e desvios padrão das variáveis hemogasométricas e pressão arterial média (PAM) em cadelas $(n=12)$ submetidas à ovariohisterectomia, prémedicadas com tramadol (GT) ou morfina (GM) e anestesiadas pelo isofluorano

\begin{tabular}{|c|c|c|c|c|c|c|}
\hline \multirow[b]{2}{*}{ Parâmetros } & \multirow[b]{2}{*}{ Grupos } & \multicolumn{5}{|c|}{ Momentos } \\
\hline & & MO & M15 & M30 & M45 & M60 \\
\hline & GM & $55 \pm 9$ & $50 \pm 14^{a}$ & $80 \pm 21^{b}$ & $64 \pm 18$ & $63 \pm 17$ \\
\hline \multirow{2}{*}{ PAM (mmHg) } & GT & $64 \pm 14$ & $55 \pm 11$ & $80 \pm 15$ & $70 \pm 13$ & $64 \pm 12$ \\
\hline & GM & $440 \pm 24$ & $442 \pm 43$ & $415 \pm 64$ & $377 \pm 97$ & $353 \pm 126$ \\
\hline \multirow{2}{*}{$\mathrm{PaO}_{2}(\mathrm{mmHg})$} & GT & $352 \pm 34$ & $381 \pm 42$ & $387 \pm 31$ & $376 \pm 41$ & $367 \pm 58$ \\
\hline & GM & $51 \pm 4$ & $52 \pm 2$ & $58 \pm 6$ & $57 \pm 6$ & $54 \pm 9$ \\
\hline \multirow{2}{*}{$\mathrm{PaCO}_{2}(\mathrm{mmHg})$} & GT & $50 \pm 6$ & $53 \pm 8$ & $55 \pm 11$ & $60 \pm 10$ & $56 \pm 10$ \\
\hline & GM & $100 \pm 0$ & $100 \pm 0$ & $100 \pm 0$ & $100 \pm 0$ & $100 \pm 0$ \\
\hline \multirow{2}{*}{$\mathrm{SaO}_{2}(\%)$} & GT & $100 \pm 0$ & $100 \pm 0$ & $100 \pm 0$ & $100 \pm 0$ & $100 \pm 0$ \\
\hline & GM & $-6,7 \pm 1,8$ & $-6,6 \pm 1,6$ & $-7,5 \pm 1,9$ & $-8,7 \pm 1,6$ & $-8,9 \pm 3,2$ \\
\hline \multirow{2}{*}{$\mathrm{DB}(\mathrm{mmol} / \mathrm{L})$} & GT & $-6,0 \pm 1,9$ & $-6,6 \pm 2,0$ & $-7,2 \pm 1,7$ & $-7,1 \pm 2,3$ & $-7,2 \pm 3,4$ \\
\hline & GM & $20,8 \pm 1,4$ & $21,2 \pm 1,3$ & $21,5 \pm 1,6$ & $20,9 \pm 1,8$ & $19,9 \pm 3,3$ \\
\hline \multirow{2}{*}{$\mathrm{HCO}_{3}^{-}(\mathrm{mmol} / \mathrm{L})$} & GT & $21,2 \pm 2,1$ & $21,3 \pm 2,5$ & $21,5 \pm 2,8$ & $22,3 \pm 3,3$ & $21,9 \pm 4,2$ \\
\hline & GM & $7,24 \pm 0,04$ & $7,23 \pm 0,03$ & $7,19 \pm 0,05$ & $7,18 \pm 0,04$ & $7,18 \pm 0,05$ \\
\hline $\mathrm{pH}$ & GT & $7,24 \pm 0,02$ & $7,23 \pm 0,03$ & $7,21 \pm 0,04$ & $7,18 \pm 0,02$ & $7,21 \pm 0,03$ \\
\hline
\end{tabular}

Letras minúsculas diferentes nas linhas indicam significância estatística, com $p<0,05$. da $f$ pelo Vti (Levitzky, 2004). Sabe-se que a morfina deprime a respiração resultando no decréscimo do VM respiratório e aumento $\mathrm{ETCO}_{2}$, (Thurmon et al., 1996). Também se sabe que o isofluorano promove diminuição do VM (Amaral, 2001). Portanto, sugere-se que os valores mais altos de Vti em GM compensaram a possível diminuição no VM provocado pela morfina associada ao anestésico inalatório isofluorano. Também se observou que os valores de $\mathrm{ETCO}_{2}$ no GM ficaram acima dos considerados normais para a espécie (35 a $45 \mathrm{mmHg}$ ), segundo Haskins (1996).

As variáveis hemogasométricas estudadas nesse trabalho não apresentaram diferenças estatísticas entre os grupos GM e GT ou dentro dos grupos, demonstrando que não são os opioides os causadores das alterações encontradas e discutidas abaixo.

Tanto no GM quanto no GT, para a $\mathrm{PaO}_{2}$ e $\mathrm{SaO}_{2}$, observaram-se médias altas. Tal fato não constituiu surpresa, visto que ambas variáveis têm relação direta com a porcentagem de oxigênio inspirado (no estudo $\mathrm{FiO}_{2}=1$ ) (Cortopassi et al., 2002). Para animais hígidos, a $\mathrm{PaO}_{2}$ prevista é de aproximadamente quatro a cinco vezes a porcentagem de oxigênio inspirado e para a $\mathrm{SaO}_{2}$, em cães,

\section{Discussão}

As alterações nas variáveis ventilométricas, que podem indicar distúrbios respiratórios levando a situações de risco para os animais, principalmente durante procedimentos cirúrgicos (Guyton e Hall, 1997; Haskins, 1996; Jones, 1996; Nunes, 2002), foram poucas, mantendo-se dentro da faixa de normalidade para a espécie canina. Esses resultados sugerem a segurança dos fármacos em relação ao sistema respiratório, o que já vem sendo descrito em estudos com humanos (Budd e Langford, 1999; Houmes et al., 1992).

O volume corrente inspirado ( $\mathrm{Vti}$ ) é o volume de ar que entra e sai das vias aéreas superiores a cada ciclo respiratório, sendo determinado pela atividade do centro de controle respiratório do cérebro e pela mecânica pulmonar do paciente (complacência e resistência respiratória) (Levitzky, 2004). Para obtenção de uma ventilação adequada, preconiza-se a utilização de um Vti de 10$15 \mathrm{~mL} / \mathrm{kg}$ (Robertson, 2004). Apesar das médias maiores no GM em M0 a M60 (Tabela1), os valores médios para Vti em ambos os grupos não ficaram aquém dos considerados normais para a espécie, visto que o peso médio dos animais ficou em $15 \mathrm{~kg}$.

Mesmo sem diferenças significativas na $f$ em GM e GT, o aumento das médias de Vti no GM pode estar relacionado com a manutenção do volume minuto (VM), que é resultado do produto são considerados normais valores acima de 90\% (Cortopassi et al., 2002). No presente estudo as médias encontradas estão dentro dessa faixa.

Ao analisar os dados do pH no GM e GT, verificaram-se médias abaixo dos valores normais para a espécie (Tabela 2). Segundo Luna (2002), os valores de $\mathrm{pH}$ variam entre 7,35 e 7,45. Esses resultados indicam provavelmente a ocorrência de um quadro compatível com acidemia nos dois grupos (acidose respiratória não compensada). Houpt (1993) e Jhonson e Morais (2007) afirmaram que maiores médias de $\mathrm{PaCO}_{2}$ proporcionam aumento de ácido carbônico e redução do $\mathrm{pH}$, o que foi encontrado nesse estudo, pois a $\mathrm{PaCO}_{2}$ normal no cão é de $40 \pm 7 \mathrm{mmHg}$ (Morgan e Mikhail, 1996) e valores acima podem estar associados à presença de hipoxemia e acidose respiratória (Haskins, 1996).

Segundo Hubbell (1991), a diminuição da ventilação durante a anestesia geral aumenta a $\mathrm{PaCO}_{2}$, diminuindo o $\mathrm{pH}$ arterial e celular. Além disso, Amaral (2001) relatou que a anestesia de cães com isofluorano provoca aumento da $\mathrm{PaCO}_{2}$, corroborando os achados desse estudo e as alterações nas médias de $\mathrm{pH}$.

Em relação à variável diferença de bases (DB), esta pode ser definida como a quantidade de base acima ou abaixo da base tampão normal e os valores negativos espelham um déficit de base ou um excesso de ácido (Evora et al., 1999). Segundo 
Luna (2002), o intervalo considerado normal para a espécie varia entre $-3 \mathrm{a}+3 \mathrm{mEq} / \mathrm{L}$. Analisando os dois grupos, em todos os momentos os valores ficaram fora dessa faixa de normalidade, sendo que isso provavelmente ocorreu em virtude da acidose respiratória não compensada, provocada pelo isofluorano, registrada em GM e GT durante o período experimental, indicando um excesso de ácido (Evora et al., 1999).

Para a PAM, observou-se diferença significativa apenas dentro do GM, porém manteve-se dentro dos parâmetros fisiológicos em ambos os grupos, considerando-se que a pressão arterial média inferior a 60 mmHg indica hipotensão (Litmman e Drobatz, 1997).

\section{Conclusões}

De acordo com a metodologia empregada e com base na análise dos resultados obtidos, pode-se concluir que ambos os opioides testados não promoveram alterações significativas nas variáveis ventilométricas quando utilizados como pré-medicação em cadelas submetidas à cirurgia de OHE e anestesiadas com isofluorano. Entretanto, em ambos os grupos, a anestesia geral inalatória causou alterações hemogasométricas importantes, culminando com acidose respiratória, o que deve levar os anestesistas a terem cautela durante a anestesia de animais já predispostos à acidema.

\section{Agradecimentos}

Os autores agradecem ao Programa de Pós-Graduação em Cirurgia Veterinária da Faculdade de Ciências Agrárias e Veterinárias - Unesp Jaboticabal, SP, pelo apoio financeiro concedido e cessão dos materiais para a execução do projeto.

\section{Referências}

AMARAL, J.L.G. (2001). Anestesia inalatória. In: YAMASHITA, A.M. et al. Anestesiologia - SAESP - Sociedade de Anestesiologia do Estado de São Paulo. 5. ed. São Paulo: Atheneu, p. 551-577.

BOSCAN, P.; PYPENDOP, B.H.; SOLANO, A.M. et al. (2005). Cardiovascular and respiratory effects of ketamine infusions in isoflurane-anesthetized dogs before and during noxious stimulation. American Journal of Veterinary Research, Schaumburg, v. 66, n. 12, p. 2122-2129.

BOZKURT, P. Use of tramadol in children. Pediatric Anesthesia, v.15, p. 1041-1047, 2005.

CORTOPASSI, S. R. G. et al. Complicações da anestesia. In: FANTONI, D. T; CORTOPASSI, S. R. G. Anestesia em cães e gatos. São Paulo: Roca, 2002, cap. 33, p. 347-361.

DOHERTY, T.; REDUA, M. A.; QUEIROZ-CASTRO, P. et al. Effect of intravenous lidocaine and ketamine on the minimum alveolar concentration of isoflurane in goats. Veterinary Anaesthesia and Analgesia, v. 34, n. 2, p. 125-131, 2007.

EVORA, P.R.B. et al. Distúrbios do equilíbrio hidroeletrolítico e do equilíbrio ácido básico- uma revisão prática. Medicina, Ribeirão Preto, v. 32, p. 451-469, 1999.

FANTONI, D. T.; MASTROCINQUE, S. Fisiopatologia e controle da dor. In: FANTONI, D. T.; CORTOPASSI, S. R. G. Anestesia em cães e gatos. São Paulo: Roca, 2002. Cap .31, p. 329-330.

FANTONI, D.T.; KRUMENERI, J.L.; GALEGO, M. P. Utilização de analgésicos em pequenos animais. Clínica Veterinária, ano v, $\mathrm{n}$. 28, p. 23-33, Set./Out., 2000.

GAYNOR, J. S. Is postoperative pain management important in dogs and cats. Veterinary Medicine, v. 3, n. 4, p. 254-257, 1997.

GÓRNIAK, S. L. Hipnoanalgésicos. In: SPINOSA, H. S.; GÓRNIAK, S.L.; BERNARDI, M. M. Farmacologia aplicada à medicina veterinária. Rio de Janeiro: Guanabara Koogan, 2002. cap.15, p.164.

GREMIÃO, I. D. F.; NASCIMENTO, J, A.; SOARES, J. H. N. et al. Redução da concentração alveolar mínima (CAM) em cães anestesiados com isofluorano associado a fentanila. Acta Scientiae Veterinariae, v. 31, n. 1, p. 13-19, 2003.

GRIEF, R. et al. Blood pressure response to thermoregulatory vasoconstriccion during isofluorane and desflurane anesthesia. Acta. Anaesth. Scand., v. 47, p. 847-852, 2003.
GROND, S.; SABLOTZKI, A. Clinical pharmacology of tramadol. Clinical Pharmacokinetics, Auckland, v. 43, n.13, p. 879-923, 2004.

GUYTON, A. C.; HALL, J. E. Transporte de oxigênio e de dióxido de carbono no sangue e em outros líquidos corporais. In: GUYTON. A. C. Tratado de fisiologia médica. 9. ed. Rio de Janeiro: Interamericana. cap. 48, p. 465-474, 1997.

HASKINS, S. C. Monitoring the anesthetized patient. In: THURMON, J. C.; TRANQUILLI, W. J.; BENSON, G. J. Lumb \& Jones' veterinary anesthesia, 3. ed. Philadelphia: Lippincott Williams \& Wilkins, cap.1, p. 3, 1996.

HOUMES, R. J.; VOETS, M. A.; VERKAAIK, A. et al. Efficacy and safety of tramadol versus morphine for moderate and severe postoperative pain with special regard to respiratory depression. Veterinary Anaesthesia and Analgesia, v. 74, n. 4, p. 510-514, 1992.

HOUPT, T. R. Equilíbrio ácido-básico. In: SWENSON, M. J.; REECE, W. O. Dukes: fisiologia dos animais domésticos. 11. ed. Rio de Janeiro: Guanabara Koogan, 1993. cap. 32, p. 549-559.

HUBBELL, J.A.E. Oxygen supplementation and ventilatory assist devices. In: MUIR, W.W.; HUBBELL, J.A.E. Equine Anesthesia (Monitory and Emergency Therapy). St Louis: Mosby Year Book, 1991, p. 401-418.

JONES, J. L. Noninvasive monitoring techniques in anesthetized animals. Veterinary Medicine, Lenexa, v. 91, n. 4, p. 326-325, 1996.

JOHSON, R.A.; MORAIS, H.A. Distúrbios ácido-básicos respiratórios. In: DiBARTOLA, S.P. Anormalidades de fluidos, eletrólitos e equilíbrio ácido-básico na clínica de pequenos animais. São Paulo: ROCA, 2007. p. 270-283.

LASCELLES, B. D.; CRIPPS, P. J.; JONES, A. et al. Postoperative central hypersensitivity and pain: the preemptive value of phetidine for ovariohysterectomy. Pain, v. 73, p. 461-471, 1997.

LEVISTZKY, M. G. Ventilação alveolar. In: São Paulo: Manole, 2004. cap. 3, p. 55-85.

Fisiologia Pulmonar.

LITMMAN, M. P.; DROBATZ, K. L. Distúrbios hipertensivos e hipotensivos. In: ETTINGER, S. J.; FELDMAN, E. C. Tratado de medicina interna veterinária: moléstias do cão e do gato. São Paulo: Manole, 1997. cap. 19, p. 126-135.

LUNA, S. P. L. Equilíbrio ácido-básico. In: FANTONI, D. T.; CORTOPASSI, S. R. G. Anestesia em cães e gatos. São Paulo: Roca, 2002. cap. 10, p. 121-139. 
ORGAN JR, G.E.; MIKHAIL, M.S. Fisiologia respiratória e anestesia. In: MORGAN JR, G.E.; MIKHAIL, M.S. Anestesiologia Clínica. 2. ed. Rio de Janeiro: Revinter. 2003. cap. 22. p. 392-421.

NUNES, N. Monitoração da anestesia. In: FANTONI, D. T.; CORTOPASSI, S. R. G. Anestesia em cães e gatos. São Paulo: Roca, 2002. cap. 6, p. 64-81.

PASCOE, P.J. Opioid analgesics. Veterinary Clinics of North America: Small Animal Practice, v. 30, n. 4, p.75 7-772, July 2000.

PYPENDOP, B. H.; SOLANO, A.; BOSCAN, P. et al.

Characteristics of the relationship between plasma ketamine concentration and its effect on the minimum alveolar concentration of isoflurane in dogs. Veterinary Anaesthesia and Analgesia, v. 34, n. 3, p. 209-212, 2007.

RADBRUCH, L.; GROND, S.; LEHMANN, K. A. A risk-benefit assessment of tramadol in the management of pain. Drug Safety, Auckland, v. 15, n. 1, p. 8-29, 1996.

REZENDE, M. L.; SANTOS, P. S. P.; NUNES, N. et al. Emprego da levomepromazina no bloqueio da arritmia induzida pela adrenalina em cães anestesiados pelo sevoflurano. Ciência Rural, v. 30, n. 3, p. 421-424, 2000.
ROBERTSON, S.A. Oxigenação e ventilação. In: GREENE, S.A. Segredos em anestesia veterinária e manejo da dor. Porto Alegre: Artmed, 2004, cap. 2, p. 31- 36.

SHEPHERD, G. M. Hypersensitivity reactions to drugs: evaluation and management. The Mount Sinai Journal of Medicine. New York, v. 70, n. 2, p. 113-125, 2003.

SHORT, E.C. Fundamentals of pain perception in animals. Applied Animal Behaviour Science, n. 59, 1998.

STEFFEY, E. P. Inhalation anesthetics. In: THURMON, J. C. et al. Lumb \& Jones veterinary anesthesia. 3. ed. Philadelphia: Williams \& Wilkins, p. 297- 329, 1996.

THURMON, J. C. et al. Preanesthetics and anesthetic adjuncts. In:_. Lumb \& Jones' veterinary anesthesia. 3. ed. Philadelphia: Lea \& Feabiger, 1996. p. 183-209.

VEIEN, M. et al. Mechanisms of nonimmunological histamine and tryptase release from human cutaneous mast cells.

Anesthesiology. Philadelphia, v. 92, n. 1, p. 1074-1081, 2000. 